\title{
Systematic literature review and meta-analysis of the efficacy of artemisinin-based and quinine-based treatments for uncomplicated falciparum malaria in pregnancy: methodological challenges
}

Makoto Saito ${ }^{1,2,3^{*}}$, Mary Ellen Gilder ${ }^{3}$, François Nosten ${ }^{2,3}$, Rose McGready ${ }^{2,3}$ and Philippe J. Guérin ${ }^{1,2}$

\begin{abstract}
Background: There is no agreed standard method to assess the efficacy of anti-malarials for uncomplicated falciparum in pregnancy despite an increased risk of adverse outcomes for the mother and the fetus. The aim of this review is to present the currently available evidence from both observational and interventional cohort studies on antimalarial efficacy in pregnancy and summarize the variability of assessment and reporting found in the review process.

Methods: Efficacy methodology and assessment of artemisinin-based treatments (ABT) and quinine-based treatments (QBT) were reviewed systematically using seven databases and two clinical trial registries (protocol registration—PROSPERO: CRD42017054808). Pregnant women in all trimesters with parasitologically confirmed uncomplicated falciparum malaria were included irrespective of symptoms. This review attempted to re-calculate proportions of treatment success applying the same definition as the standard WHO methodology for non-pregnant populations. Aggregated data meta-analyses using data from randomized control trials (RCTs) comparing different treatments were performed by random effects model.

Results: A total of 48 eligible efficacy studies were identified including 7279 treated Plasmodium falciparum episodes. While polymerase chain reaction (PCR) was used in 24 studies for differentiating recurrence, the assessment and reporting of treatment efficacy was heterogeneous. When the same definition could be applied, PCR-corrected treatment failure of $\geq 10 \%$ at any time points was observed in 3/30 ABT and 3/7 QBT arms. Ten RCTs compared different combinations of ABT but there was a maximum of two published RCTs with PCR-corrected outcomes for each comparison. Five RCTs compared ABT and QBT. Overall, the risk of treatment failure was significantly lower in ABT than in QBT (risk ratio 0.22, 95\% confidence interval 0.07-0.63), although the actual drug combinations and outcome endpoints were different. First trimester women were included in 12 studies none of which were RCTs of ABT.
\end{abstract}

Conclusions: Efficacy studies in pregnancy are not only limited in number but use varied methodological assessments. In five RCTs with comparable methodology, ABT resulted in higher efficacy than QBT in the second and third

\footnotetext{
*Correspondence: makoto.saito@wwarn.org

${ }^{2}$ Centre for Tropical Medicine and Global Health, Nuffield Department

of Medicine, University of Oxford, Old Road Campus, Roosevelt Drive,

Oxford OX3 7FZ, UK

Full list of author information is available at the end of the article
} 
trimester of pregnancy. Individual patient data meta-analysis can include data from observational cohort studies and could overcome some of the limitations of the current assessment given the paucity of data in this vulnerable group.

Keywords: Malaria, Pregnancy, Efficacy, Artemisinin, Quinine, Methodology, Review

\section{Background}

Approximately $60 \%$ of all pregnancies worldwide take place in malaria endemic areas, leading to 125 million pregnant women at risk of malaria every year [1]. Malaria in pregnancy, regardless of whether it is clinically symptomatic or not, has been reported to be associated with a higher risk of preterm birth, low birth weight for gestational age, miscarriage, stillbirth and maternal anaemia [2-5]. These adverse outcomes lead to a higher risk of perinatal mortality and maternal mortality in areas with low or declining malaria prevalence compared to high transmission areas because of lower levels of premunition $[6,7]$.

In order to mitigate these adverse effects, efficacious treatments need to be clearly identified for pregnant women. However, several factors have limited the available evidence on anti-malarials efficacy during pregnancy. Pregnant women are usually excluded from randomized control trials (RCTs) of new anti-malarials mainly because of concerns about the safety for the fetus. Safety concerns were particularly critical for artemisinin derivatives, as fetal resorption was observed in animal studies [8-13]. Quinine-based treatment (QBT) is still recommended as the first-line treatment for uncomplicated falciparum malaria in the first trimester [14], despite limited clinical data on its safety in the first trimester [15, 16]. Quinine's poor side effect profile and long treatment course of 5-7 days make it an undesirable choice for patients [15-17]. Recently, data from prospective observational cohort studies suggest that artemisinin use in the first trimester did not increase the risks of stillbirth or congenital abnormality compared to quinine [3, 18-21], bringing the question of comparative efficacy into center stage.

Efficacy of anti-malarial drugs for treating uncomplicated malaria in non-pregnant patients has traditionally been assessed over a fixed follow-up period set by the current World Health Organization (WHO) recommendations at 28-42 days [22]. However, this fixed period does not accommodate the pregnant condition as the placenta may become or remain parasitized (placental sequestration) after treatment completion [23]. There are currently no standard guidelines on parasitological efficacy studies in pregnancy [24].
With this in mind, this systematic literature review aims to update the currently available efficacy data of artemisinin-based treatments (ABT) and QBT from both observational and interventional cohort studies in all trimesters with uncomplicated falciparum malaria. Methodological challenges will also be summarized to improve future efficacy studies in pregnancy.

\section{Methods}

A systematic literature review following PRISMA statement [25] was conducted to identify studies measuring the efficacy of ABT or QBT in pregnant women with parasitologically confirmed uncomplicated falciparum malaria, regardless of trimester or clinical symptoms. Seven databases (MEDLINE, Embase, Global Health, Cochrane Library, Scopus, Web of Science and LILACS) and two clinical trial registries (ICTRP and ClinicalTrial. gov) were used. This review is registered to PROSPERO (CRD42017054808), and the search terms and conditions are available in Additional file 1.

The search (conducted 9 July 2016-10 January 2017) combined five components: malaria; pregnancy; treatment or names of anti-malarial drugs; study design (interventional or observational cohort studies); and outcome types (efficacy) without limitation on publication year or language. Two reviewers (MS and MEG) assessed eligibility independently, and discrepant results were resolved by a second assessment.

Both interventional and observational cohort studies were included. Studies without any active follow-up in the first 28 days were excluded. Studies with fewer than ten pregnant women were excluded, as they could not be included in further meta-analyses. Systematic reviews on the anti-malarial treatment in pregnancy $[15,26-29]$ were checked for any other possible missing articles that should be included.

Uncomplicated Plasmodium falciparum malaria was defined as malaria infection without features of severe malaria [14]. Pregnancy was described by trimesters: the first as < 13 completed weeks, the second as from 14 weeks to 27 completed weeks, and the third from 28 weeks until delivery.

After screening, the following data were extracted: demographic information of study (year, country, study 
design, study drugs and eligibility criteria), availability of outcome assessment (clinical outcomes, parasitological outcomes) and the methodology of assessment of variables (definition of treatment success and statistical method). Information was sought from published articles, clinical trial registry and protocols if available. Missing information was supplemented by personal correspondence to authors of the original studies if possible. This review describes the methodology of assessment and reporting, and summarizes the reported efficacy results. Comparisons were made only if the same or similar assessment methods were used.

The WHO recommends that treatment failure and completion of follow-up without treatment failure (adequate clinical and parasitological response, ACPR) be used as efficacy endpoints in non-pregnant populations, and that results be expressed as the proportion of ACPR or the cumulative success using Kaplan-Meier survival analysis. WHO advises patients with polymerase chain reaction (PCR)-confirmed reinfection with $P$. falciparum, infection with other malaria species, or loss to followup to be censored on the day of these events in the survival analysis and to be excluded from the proportional ACPR analysis [22]. Patients without PCR results or with indeterminate PCR results are to be excluded from both PCR-corrected survival analysis and PCR-corrected proportional ACPR analysis.

For the comparability across studies, PCR-corrected proportional ACPR was recalculated using the WHO guideline for non-pregnant populations [22], with the exception of cases with non-falciparum malaria infection which could not be excluded without detailed individual patient data. When the number of patients with ACPR was unavailable, it was estimated from the presented results and included for reference. PCR-corrected ACPR of $90 \%$ was used as the cut-off value to judge whether the treatment was satisfactory or not [14]. The 95\% confidence interval $(\mathrm{CI})$ for the proportion was calculated by the Wilson method [30]. Random effects meta-analyses using aggregated results from RCTs were conducted if there were more than two RCTs. Heterogeneity was assessed using $\mathrm{I}^{2}$ [31]. If there was no treatment failure, a continuity correction was made by adding 0.5 for both treatment success group and failure group. The quality of data at both the study level and each outcome level was assessed using the GRADE system [32]. GRADEpro was used for making an evidence profile table [33]. For assessing publication bias, funnel plots of the proportions were drawn with $\log$ odds for the $\mathrm{x}$-axis and study size (number of patients in each treatment arm) for the $y$-axis [34]. Asymmetry was judged visually, as formal statistical tests have not been developed for this method [34]. For odds ratios, Egger's test was used for checking the asymmetry of funnel plots [35]. STATA MP 14.2 (Stata Corp, Texas, US) was used for the statistical analyses.

\section{Results}

A total of 48 study cohorts assessing treatment efficacy for uncomplicated falciparum malaria in pregnancy were identified (see Additional file 2) evaluating at least 7279 episodes of parasitologically confirmed uncomplicated falciparum. Studies based on the same cohort were considered as a single study. Forty-one studies were published, five presented at conferences, and two were registered on a public trials database but not yet published. Twenty-six studies (54\%) were from sub-Saharan Africa, 19 (40\%) from Southeast Asia, two (4\%) from Latin America and one (2\%) from India (Fig. 1). As of January 2017, all unpublished registered trials assessing anti-malarial efficacy in pregnancy were either completed $(\mathrm{n}=2)$ or withdrawn $(\mathrm{n}=3)$ : two trials (NCT00331708, NCT01082731) were terminated because of slow recruitment, and the other (NCT01082718) was withdrawn before enrolment.

The study designs comprised 22 RCTs comparing two or more treatment regimens [17, 36-57], 10 pharmacokinetic (PK) studies including clinical outcome assessment [58-68], six single arm interventional studies [69-74] and 10 observational cohort studies [75-85] (Table 1 and Additional file 3). Blinding varied with one double-blind study [54] and one study using placebo but with unspecified blinding [17] (see Additional file 4). In seven studies, assessors and laboratory staff $(n=2)[45,48]$ or only laboratory staff (e.g. microscopists) $(\mathrm{n}=5)[44,46,50,52$, 57] were blinded.

\section{Study drugs}

Fourteen studies included women treated with QBT, 40 studies included ABT, and six studies included both.

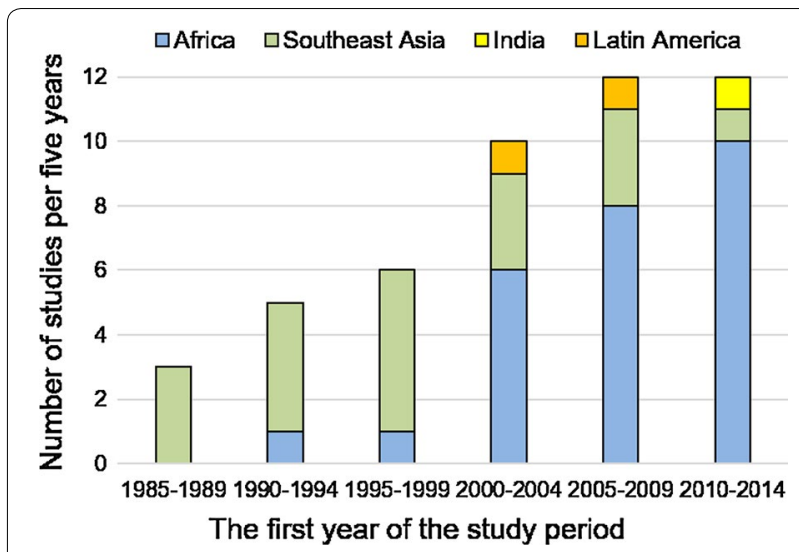

Fig. 1 Number of studies on treatment efficacy of malaria in pregnancy per 5 years (1985-2016). The first year of study period was used for categorization. No studies were identified after 2015 
Altogether, 6244 and 1035 episodes were treated with ABT or QBT, respectively.

Quinine was administered at $30 \mathrm{mg} / \mathrm{kg} /$ day for 7 days in nine studies $(64 \%, 9 / 14)$. The other five studies gave similar dosing with slight variations: one study administered for 7 - or more-days depending on the clinical condition [39]; one study started with intravenous quinine until oral therapy was tolerated [36]; and one study compared standard dose with lower dose $(20 \mathrm{mg} / \mathrm{kg} /$ day $)$ for 7 days [43]; one study administered $30 \mathrm{mg} / \mathrm{kg} /$ day for 5 days [17]; one study gave $1800 \mathrm{mg} /$ day for 7 days [37]. Artesunate was given at $10-16 \mathrm{mg} / \mathrm{kg} /$ course $(600-$ $900 \mathrm{mg} /$ course) over 3-7 days except in two PK studies: one PK study administered $200 \mathrm{mg}$ of artesunate once [60], and the other PK study administered total $28 \mathrm{mg} / \mathrm{kg}$ over 7 days (1 day intravenously and 6 days orally) [61]. Artesunate-amodiaquine (ASAQ) was given as a fixed dose combination in four studies $(57 \%, 4 / 7)$ and as a nonfixed dose combination with $30 \mathrm{mg} / \mathrm{kg}$ of amodiaquine in three studies $(43 \%, 3 / 7)$. Artesunate-mefloquine (ASMQ) was given as a fixed dose combination in four studies $(36 \%, 4 / 11)$ and as a non-fixed dose combination with $25 \mathrm{mg} / \mathrm{kg}$ of mefloquine in six studies $(55 \%, 6 / 11)$. Non-fixed dose combination of an unknown dose was assumed to be used in one study [81]. Artemether-lumefantrine (AL) was administered for 3 days $(88 \%, 14 / 16)$ except two studies: one for 4 days [57] and the other for 5 days [53]. Supervision of treatments also varied (see Additional file 5).

Five RCTs compared QBT and ABT but only two of them used the same ABT (i.e. non-fixed dose combination ASMQ) [39-42, 48]. Ten RCTs compared different combinations of ABT [38, 45, 49, 50, 52-57] (see Additional file 6). Two RCTs compared different regimens of QBT $[17,43]$, two compared quinine versus non-ABT $[36,37]$ and three compared ABT versus non-ABT (e.g. sulfadoxine-pyrimethamine (SP) or chlorproguanil-dapsone) $[44,46,47]$.

\section{Inclusion criteria}

The demographic background of patients is summarized (see Additional file 7). Asymptomatic patients were included in 55\% (12/22) of RCTs and 50\% (13/26) of non-RCTs. Two of them included only asymptomatic women $[52,60]$. Fifteen studies $(31 \%, 15 / 48)$ intentionally included patients who failed previous treatment, but only three of them were RCTs $[38,43,45]$.

Overall, twelve studies $(25 \%, 12 / 48)$ included first trimester women (see Additional file 3), describing at least 599 parasitologically confirmed first-trimester malaria episodes treated with ABT $(\mathrm{n}=108)$ or QBT $(\mathrm{n}=491)$. Only one RCT intentionally included first trimester women; it was published in 1990 and compared quinine versus mefloquine [37].

In 26 studies from Africa, three studies gave two doses of intermittent preventive treatment in pregnancy (IPTp)-SP [54, 63, 66], and one study gave three doses [84]. One study reported the percentage of women who received IPTp [83]. IPTp-SP was not administered during the whole pregnancy in one study [48] and during the study period in two other studies [50,52]. In one study, enrolled patients systematically received the study drug 4 weeks after the initial treatment instead of IPTp-SP [44].

\section{Assessment of treatment efficacy Duration of follow-up}

The primary endpoint for assessing anti-malarial efficacy varied: less than 28 days $(n=2)$, day $28(n=18)$, day 42 $(\mathrm{n}=10)$, day $56(\mathrm{n}=2)$, day $63(\mathrm{n}=5)$ and until delivery $(\mathrm{n}=10)$ (Table 1). The duration of follow-up was unclear for one conference abstract [81]. After the primary endpoint of 28-63 days, a further 14 studies continued assessing parasitaemia until delivery but with different schedules and five other studies did not continue but assessed parasitaemia only at delivery.

\section{Methodology of reporting efficacy}

For handling treatment failure, the majority of the studies $(96 \%, 46 / 48)$ followed WHO standards for non-pregnant populations, except two studies which excluded cases who developed severe malaria after the treatment (i.e. early treatment failure) from the final result $[40,47]$.

Twenty-seven studies $(56 \%, 27 / 48)$ showed only the proportional ACPR or the number of patients with ACPR (or failure). Ten studies [40-42, 45, 57, 61, 62, 75, 77, 82] derived cumulative success (or failure) by survival analysis instead of or in addition to proportional ACPR. Five other studies [44, 48, 50,54, 68] used survival analysis to compare the different groups, but the proportional ACPR was presented as the point estimates. Two studies did not report ACPR but reported time to parasite clearance based on daily parasitaemia measurements [36] or time to recurrence [78]. Information was not available from four unpublished studies.

PCR was used in 24 studies $(50 \%, 24 / 48)$ for differentiating recurrences (Table 1), although in one of the studies PCR results were considered not to be reliable because of technical problems [52]. Thirteen studies $(27 \%, 13 / 48)$ used three standard molecular markers (i.e. $m s p-1, m s p-2$ and glurp). Three of the studies adopted sequential genotyping strategy $[48,50,63]$, while the remaining ten studies ran all three markers for all recurrences. Four studies $(8 \%, 4 / 48)$ used one of the three markers $[44,46,65,68]$. 


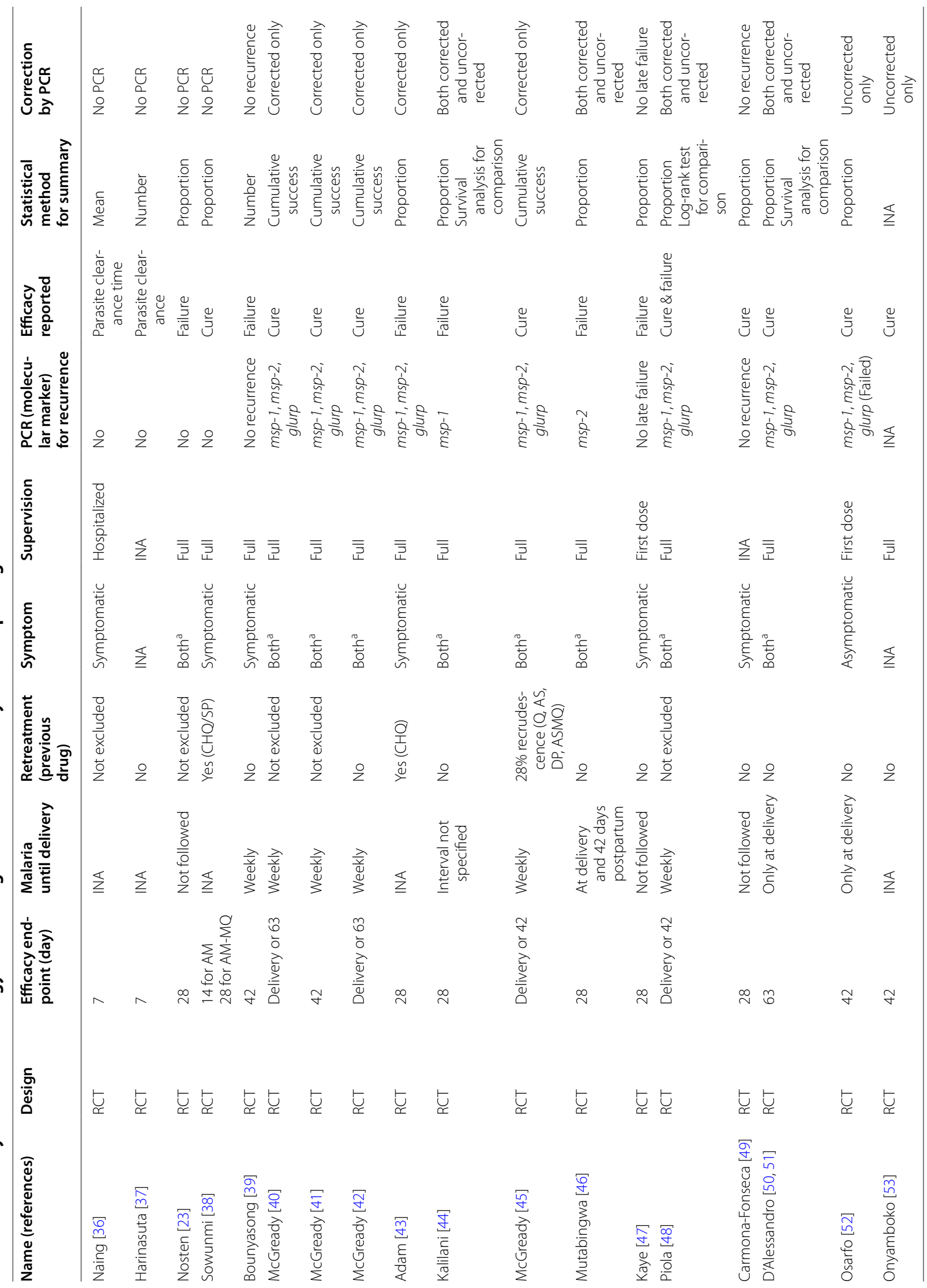




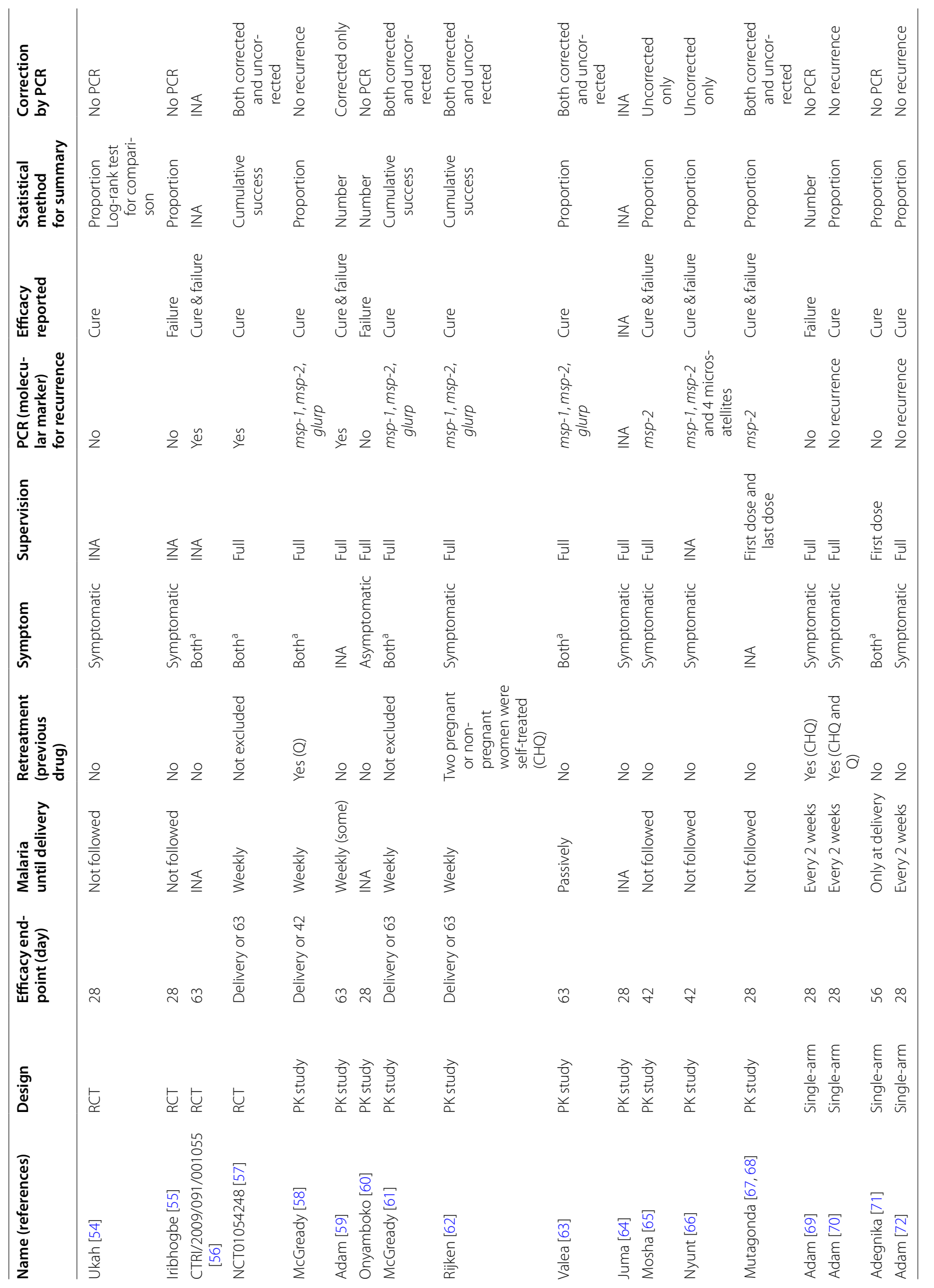




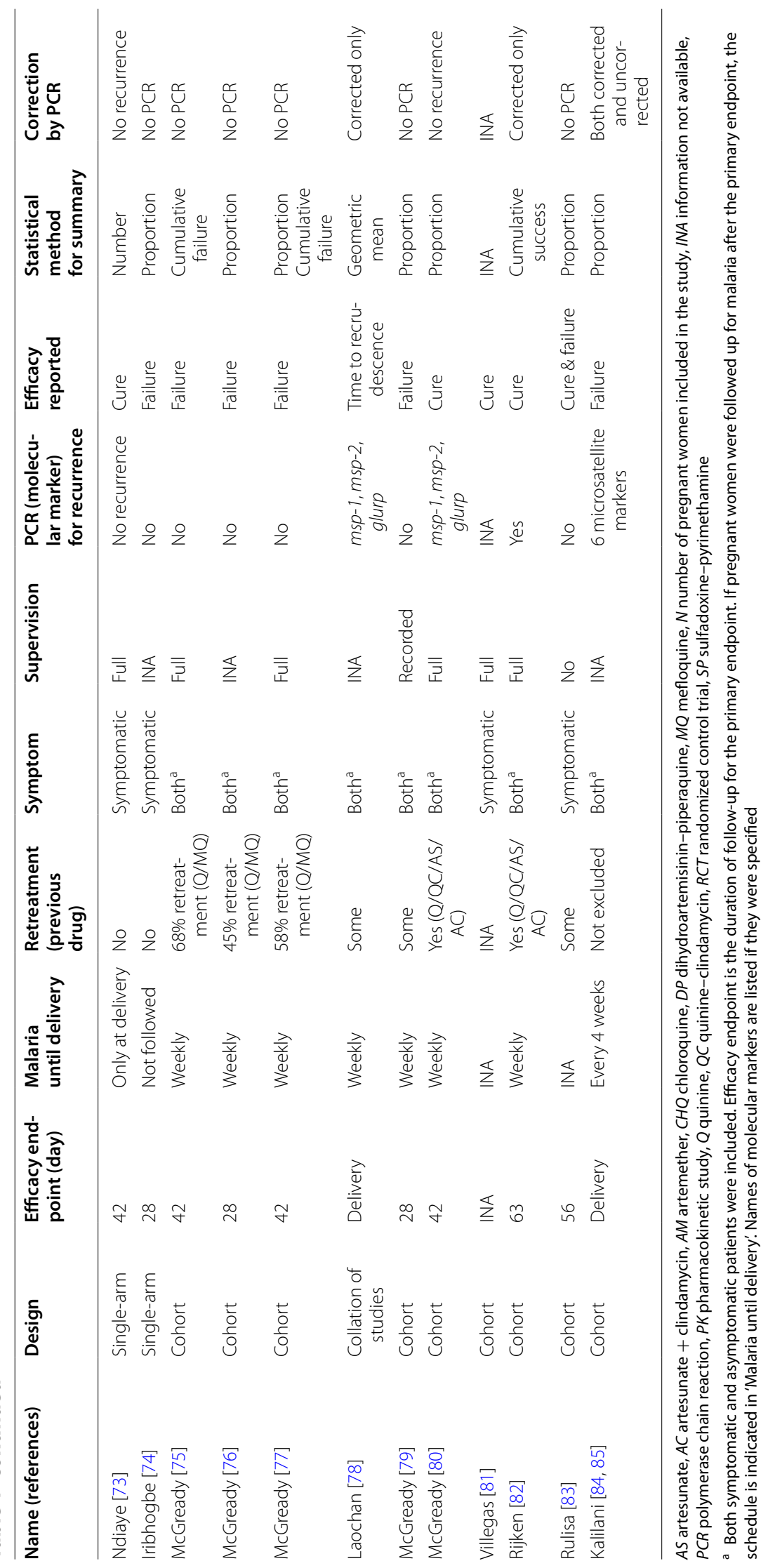


Two studies $(4 \%, 2 / 48)$ used six microsatellite markers $[66,85]$. Two published studies and two registered trials $(8 \%, 4 / 48)$ did not specify the details of molecular markers $[56,57,59,82]$. An additional six studies $(13 \%, 6 / 48)$ did not observe any late treatment failure [39, 47, 49, 70, $72,73]$, so a total of $29(60 \%, 29 / 48)$ studies contained adequate information to obtain PCR-corrected results.

There was substantial heterogeneity in how reinfections and indeterminate PCR results were handled in deriving proportional ACPR. WHO recommends excluding these cases from proportional ACPR calculations in non-pregnant populations [22]. Reinfection was excluded except in two studies in which reinfections were included as treatment success $[44,50]$. One study did not clearly report whether there were any reinfections [43]. Two studies excluded indeterminate outcomes from the calculation [48, 50], and one of them also presented results with imputation for indeterminate cases for reference [50]. Two studies using survival analysis regarded indeterminate results as recrudescence as the worst case scenario $[40,82]$. One study did not explain how they dealt with missing PCR results [46]. For proportional ACPR, no studies clearly excluded patients infected with other species of malaria before recurrence of falciparum, as recommended by the WHO non-pregnant guideline. Only one study presented results of a model which censored patients infected with Plasmodium vivax before recurrence of $P$. falciparum, and this result was provided as additional information and not the primary analysis (which did not account for P. vivax infection) [45].

Gametocyte carriage is summarized in Additional file 8. Interruption of treatment and associated adverse symptoms are summarized in Additional file 5 .

\section{Fever and parasite clearance}

Fever was assessed daily or more frequently in 27 studies $(56 \%, 27 / 48)$ and time to fever clearance was reported in 12 studies $(25 \%, 12 / 48)$. Prevalence of clearance of fever at fixed points (on day 2, 3 or 4) was reported in other 12 studies $(25 \%, 12 / 48)$. Blood smear was assessed for parasitaemia daily or more frequently at least for the first 3 days in 30 studies $(63 \%, 30 / 48)$ and time to parasite clearance was reported in 16 studies $(33 \%, 16 / 48)$. The proportion of women who cleared parasitaemia at fixed points (i.e. by $12 \mathrm{~h}$, on day 1,2 or 3 ) was reported in another 14 studies $(29 \%, 14 / 48)$.

The variability of reporting parasite and fever clearance limited comparisons across studies. In five RCTs comparing $\mathrm{ABT}$ and $\mathrm{QBT}$, three and five RCTs reported the comparison of fever clearance and parasite clearance, respectively. One RCT reported that the mean time to fever clearance was significantly shorter by ASMQ than quinine (4.47 days vs 8.04 days, $\mathrm{p}<0.001$ ), although this time was from the appearance of fever according to the patients' history rather than from the treatment [39]. Another two studies reported no difference in the proportion of febrile patients or the proportion of fever clearance by day 2 or 3 between ABT and QBT $[42,48]$. On the other hand, all five RCTs reported faster parasite clearance by ABT than by QBT. The mean or median time to parasite clearance was shorter by ABT than by quinine in two RCTs $[39,42]$. Similarly, the proportion of parasite clearance by $48 \mathrm{~h}[40,41]$ and negative parasitaemia on day 2 [48] were higher with $\mathrm{ABT}$ than QBT, although this difference was not observed on day 3 [48].

Between different ACTs, lower parasite clearance on day 1 was reported with AL compared to ASAQ, ASMQ and dihydroartemisinin-piperaquine (DP), although this was not significant on day 2 [50]. Other two RCTs also showed no difference in parasite clearance on day 3 between AL and ASAQ [54,55]. The median or mean time to fever and parasite clearance were not different between artesunate monotherapy and AL [45], and between intramuscular artemether with or without mefloquine, respectively [38].

\section{Summary of reported ACPR}

PCR-corrected treatment failure of $\geq 10 \%$ at any time points was observed in $3(10 \%, 3 / 30)$ ABT arms and 3 (43\%, 3/7) QBT arms (Figs. 2, 3). On day 28, two study arms had PCR-corrected treatment failure of $\geq 10 \%$. One of them used a lower dose quinine $(10 \mathrm{mg} / \mathrm{kg}$ twice daily rather than thrice daily) [43]. In the other study which reported the low efficacy of AL, 33\% of the enrolled patients were under retreatment, having failed previous treatment [45]. If retreatments were excluded, the cumulative success of AL on day 42 was $>90 \%$ [45]. Other study arms with PCR-corrected treatment failure of $\geq 10 \%$ were observed on day 63 (one quinine arm [40] and one artesunate monotherapy arm [61]) and until delivery (one quinine arm [42] and one AL arm [85]). The funnel plots for PCR-corrected ACPR on ABT and QBT did not show apparent asymmetry (see Additional file 9).

PCR-uncorrected ACPR was available in 14 QBT arms and $47 \mathrm{ABT}$ arms (see Additional file 10). PCR-uncorrected ACPR at different time points was available in some studies, and if it was not reported on day 28 but was $>80 \%$ on day 42,63 or at delivery, it was assumed to be $>80 \%$ on day 28 . Although different levels of the risk of reinfection due to the time and location complicate comparison between studies, at least 50\% (7/14) of QBT arms showed $\leq 80 \%$ protection within 28 days while at least 79\% (37/47) of ABT arms showed > 80\% protection within 28 days. Two ABT arms reporting $\leq 80 \%$ protection within 28 days were both conducted in high endemic areas and used AL or artesunate + SP $[44,74]$. PCR-uncorrected ACPR on day 28 was not available but 

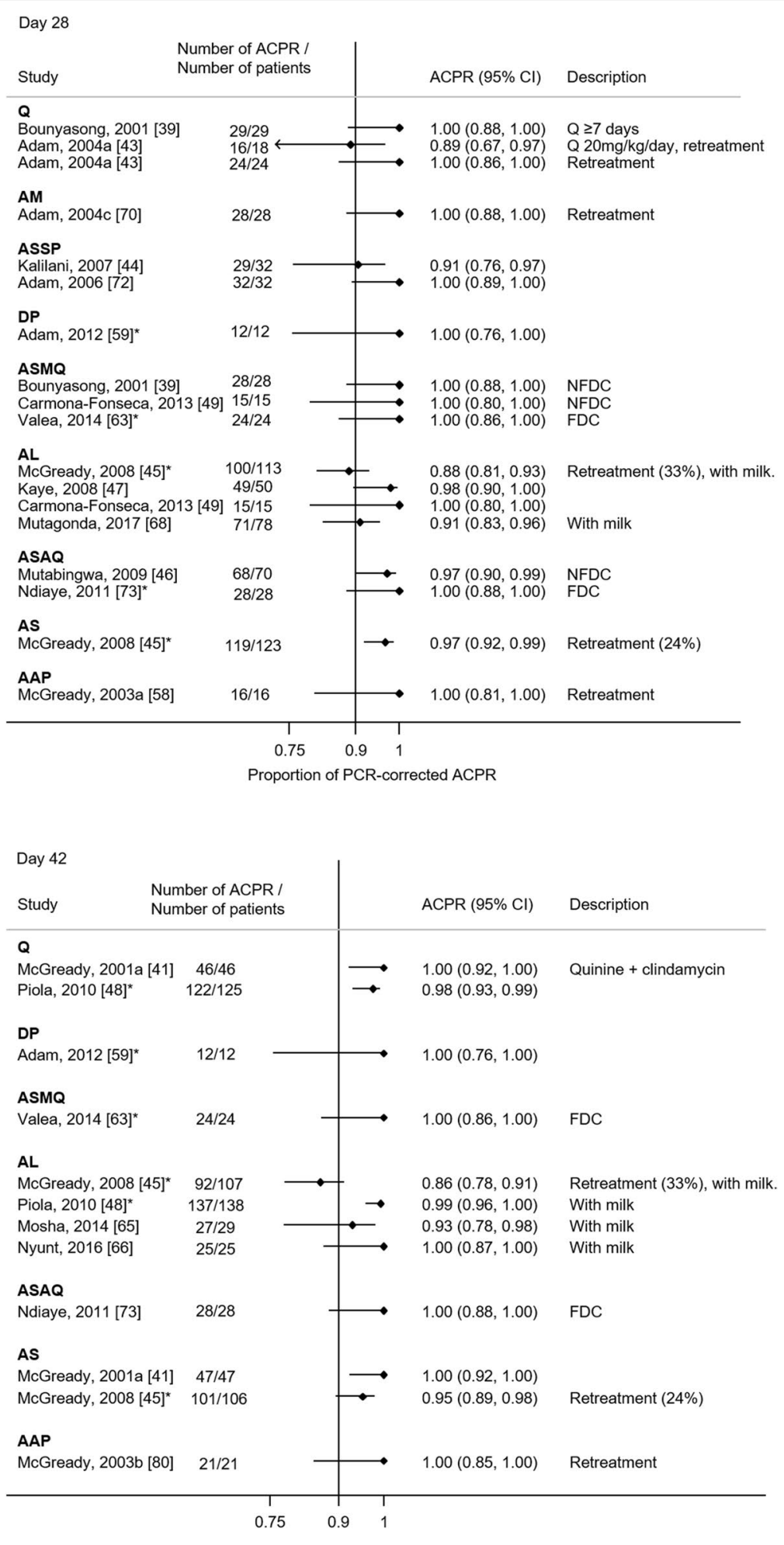

Proportion of PCR-corrected ACPR

Fig. 2 The PCR-corrected proportional adequate clinical and parasitological response (ACPR) for each study. ACPR are shown by treatment group and the duration of follow-up (i.e. day 28, 42, 63 and at delivery) with $95 \%$ confidence intervals. ${ }^{*}$ ACPR at later follow-up day is available. AAP artesunate + atovaquone-proguanil, $A C$ artesunate + clindamycin, $A L$ artemether-lumefantrine, $A M$ artemether, $A S$ artesunate, $A Q$ amodiaquine, $D P$ : dihydroartemisinin-piperaquine, FDC: fixed dose combination, MQ mefloquine, NFDC non-fixed dose combination, $Q$ quinine, $Q C$ quinine + clindamycin, SP sulfadoxine-pyrimethamine 


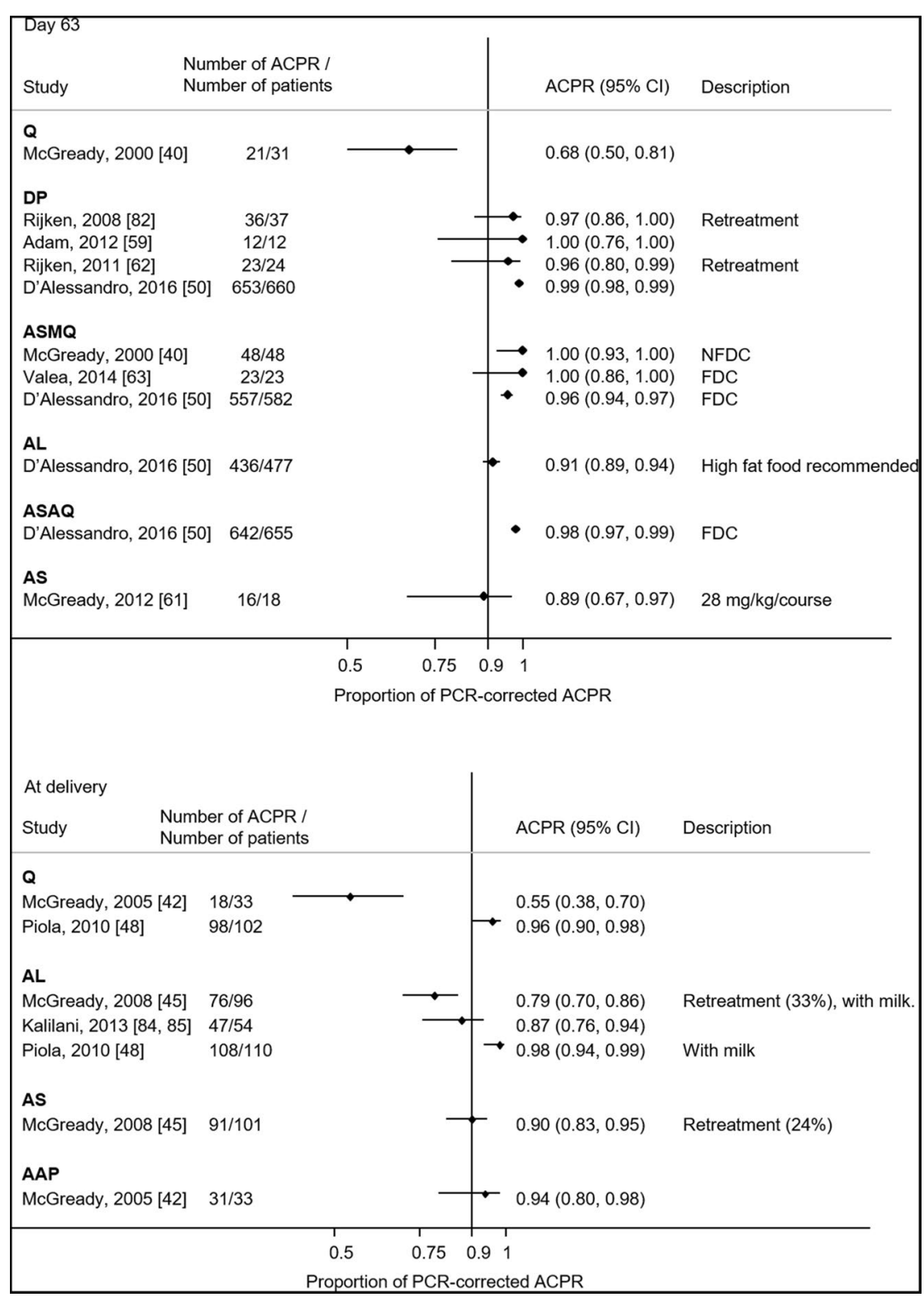

Fig. 3 The PCR-corrected proportional adequate clinical and parasitological response (ACPR) for each study (continued). ACPR are shown by treatment group and the duration of follow-up (i.e. day 28, 42, 63 and at delivery) with 95\% confidence intervals. AAP artesunate + atovaquone-proguanil, $A C$ artesunate + clindamycin, AL artemether-lumefantrine, $A M$ artemether, $A S$ artesunate, $A Q$ amodiaquine, DP: dihydroartemisinin-piperaquine, FDC: fixed dose combination, MQ mefloquine, NFDC non-fixed dose combination, Q quinine, QC quinine + clindamycin, SP sulfadoxine-pyrimethamine

it was $\leq 80 \%$ after day 28 in other eight arms from six studies $[45,50,61,75,77,82]$. ABT went on to provide protection in $>80 \%$ of patients at 42 days in at least $73 \%$ $(22 / 30)$ of the study arms which followed for 42 days or longer.

\section{PCR-corrected ACPR comparing different treatments}

Overall, the risk of treatment failure in the five RCTs available for meta-analysis was significantly lower in patients treated with ABT compared to QBT (risk ratio 0.22 , 95\% confidence interval 0.07-0.63) (Fig. 4), although the compared treatments and methodologies differed (see Additional file 11). This increased risk of failure with QBT was prominent when the patients were followed up longer. There was no evidence for asymmetry of the funnel plot suggesting publication bias $(p=0.7)$ (see Additional file 12). None of these five RCTs included pregnant women in the first trimester. 


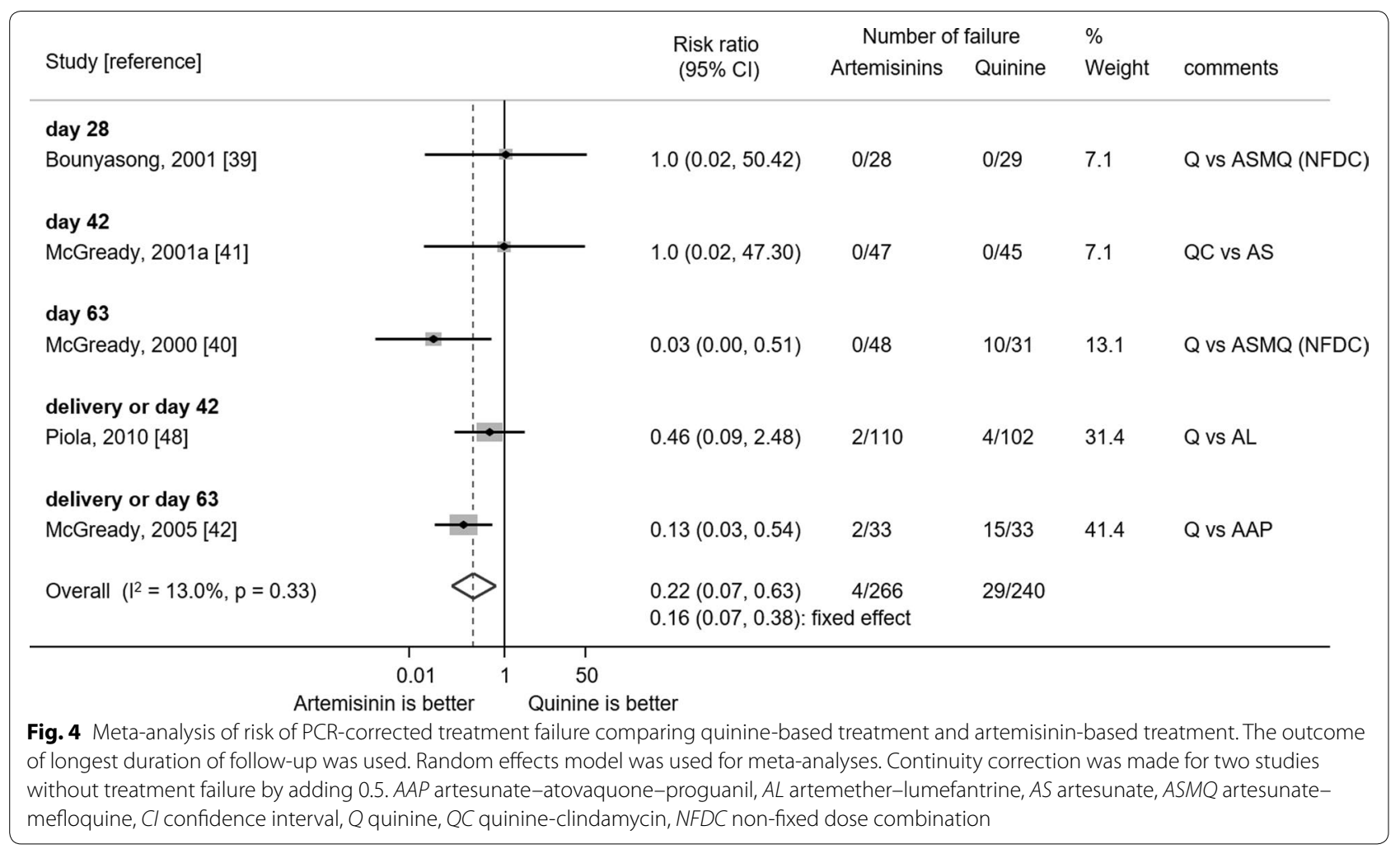

The limited number of available studies with PCRcorrected outcome comparing different ABTs precluded aggregated data meta-analysis. One RCT reported that PCR-corrected ACPR on day 63 was lower for AL than DP, ASAQ and ASMQ, although the absolute difference was $<5 \%$ [50]. Lower efficacy of AL than artesunate for 7 days was also reported when used for treating recrudescent infections [45].

\section{Time to recrudescence}

Recrudescence in pregnancy occurred after day 28 or 42, the fixed follow-up periods recommended by WHO for non-pregnant populations. In one study, one-third of the recrudescence following AL or artesunate monotherapy occurred after day 42 [45], and four studies reported recrudescence after 100 days with a maximum of 138 days [42, 44, 48, 78]. These long intervals were reported from both sub-Saharan Africa and Southeast Asia.

\section{Risk factors for recrudescence in pregnancy}

Baseline characteristics were assessed as risk factors of parasite clearance $(n=1)$ [36], recrudescence $(n=2)$ $[51,65]$ and recurrence $(\mathrm{n}=3)[52,67,71]$. One study reported gestation (early second trimester) to be associated with faster parasite clearance than late second trimester but not fever clearance [36]. Gravidity was not associated with parasite or fever clearance [36].
For recrudescence, higher baseline parasitaemia and younger maternal age were associated with higher risk in one study [51], while the other study did not find significant association with parasitaemia [65]. Gestational age $(n=2)[51,65]$, gravidity $(n=1)$ [51], haemoglobin concentration $(n=1)$ [51], body mass index $(n=1)$ [65] and symptomatic infection $(\mathrm{n}=1)$ [51] were not significant risk factors of recrudescence.

For recurrence, higher baseline parasitaemia [71], younger age [71] and lower haemoglobin concentration [67] each were associated with higher risk in one of the studies, while the other two studies did not show the associations with parasitaemia $[52,67]$, age $[52,67]$ or baseline haemoglobin concentration $[52,71]$. Lower body weight was shown to be associated with higher risk of recurrence [71]. Gestational age $(n=3)[52,67,71]$, gravidity $(\mathrm{n}=3)[52,67,71]$, parity $(\mathrm{n}=1)$ [71], body temperature $(n=1)$ [71] were not associated with the risk of recurrence.

The risk of recrudescence was higher when the drugs were used for retreatment (artemisinins [77] and quinine [76]) or recrudescence (AL) [45] than for novel infection.

\section{Placental malaria}

Placental malaria was assessed in 14 studies $(29 \%, 14 / 48)$ in various ways (see Additional file 13). Placental histopathology is the gold standard and was examined in at 
least six studies $[39,44,48,50,57,84]$, but the reporting and interpretation of the results varied with at least four different definitions [86-89]. The prevalence of placental malaria after treatment ranged from $<10 \%[45,48,62$, 80 ] to $44.8 \%$ [44], and was not different among different ABTs $[50,52]$ or between AL and quinine [48].

\section{Congenital malaria}

The peripheral blood of the newborn was assessed for malaria in six studies, of which three assessed all newborns systematically at delivery and reported the results $[38,45,48]$. Another two studies reported congenital malaria as a possible reason for neonatal deaths but did not report whether they tested for congenital malaria systematically $[40,42]$. One further study assessed congenital malaria but did not report the results [63]. No studies specified further parasitological monitoring of infants for congenital malaria diagnosed after the perinatal period.

\section{Discussion}

This review revealed the variability of study design, drugs, treatment regimens for the same drug, inclusion criteria, determination of parasitological efficacy, follow-up duration and detection of parasites in mother, placenta and newborn at delivery. The design of studies to assess the efficacy of anti-malarials during pregnancy is not standardized, and investigators are using varied adapted versions of the protocol for the non-pregnant populations currently recommended by WHO [22].

The efficacy of ABT was generally satisfactory with ACPR of $>90 \%$, although aggregated efficacy results are limited by heterogeneous methods as well as differences in patient immunity, background, symptoms, time and study site. If aggregated results are used, only a maximum of two RCTs was available for each comparison. Slightly lower efficacy of AL than other ABTs was reported in two studies $[45,50]$, and this may be explained by differences in the pharmacokinetics and pharmacodynamics of lumefantrine in pregnant women [45, 48, 66, 67, 90-93]. On the Thailand-Myanmar border, the proportion of PCR-corrected ACPR of AL was markedly low in a study of pregnant women $(<85 \%)$ [45] and not comparable to that $(>95 \%)$ reported from studies in non-pregnant populations [94, 95]. Monitoring of efficacy in pregnancy remains a useful assessment in its own right. Optimal dosing for pregnant women should be sought and extending treatment course can be an option to improve the efficacy [96]. The results of two studies assessing longer duration and higher doses of $\mathrm{AL}[53,57]$ are awaited.

The available data implied QBT has two major but not unexpected drawbacks compared to ABT (see Additional file 11): lower treatment efficacy and lower adherence.
In at least 50\% (7/14) of the study arms, one in five pregnant women or more suffered another episode of malaria parasitaemia (either recrudescence or reinfection) within 28 days after treatment by quinine. Although these studies were conducted mostly in Southeast Asia, the risk of recurrence of malaria is expected to be even higher in the high endemic areas in sub-Saharan Africa considering the short half-life of quinine. This finding is important as repetitive malaria infections during pregnancy increase adverse effects of miscarriage, small for gestational age and preterm birth cumulatively $[18,97]$. Adherence to a 7-day treatment outside of a clinical trial context is difficult for pregnant women due to the common side effect of tinnitus, which is particularly intolerable in the first 4 months of pregnancy when morning sickness peaks [17]. Adding clindamycin to quinine might provide satisfactory efficacy equivalent to ABT, but it will not overcome the poor adherence.

Several components of efficacy evaluation should be standardized for future studies. Duration of the followup period needs to be optimized for pregnant women considering physiological variations related to pregnancy [15, 23, 40, 78]: placenta sequestration [98]; different drug metabolism and distribution [99, 100]; and altered immunity profile [98]. The current WHO recommendation of follow-up for 28-42 days [22] is based on the fact that the proportion of recrudescence after 28-42 days is negligible in the non-pregnant populations [101]. However, recrudescence in pregnancy was relatively commonly observed after 42 days, demonstrating the need for prolonged follow-up in pregnancy studies. In the small number of studies that have collected relevant data, recrudescence even after 100 days has been reported in pregnant women both in Africa [44, 48, 102] and Southeast Asia [42, 78]. The duration of parasitological follow-up after delivery also needs to be considered when delivery occurs before the end of the recommended follow-up.

Two problems were encountered with proportional ACPR for pregnancy studies. The lack of a standard led to inconsistent handling of reinfections in proportional ACPR calculations making comparisons across studies difficult. In non-pregnant populations, WHO recommends excluding those with reinfection or indeterminate PCR results from the calculation of PCR-corrected proportional ACPR [22]. However, higher proportions of reinfection and lost-to-follow-up are anticipated if longer follow-up is recommended for pregnant women. If proportional ACPR is calculated, much information will be lost. Survival analysis with censorship of these cases is likely to be a preferable approach for pregnancy studies [103]. 
Data on efficacy in the first trimester are limited to information from observational studies, precluding comparison of the outcomes using aggregated results. In addition, the published results were rarely presented separately by trimester, and the methods and quality of gestational age assessment were limited [104].

Finally, and importantly, it needs to be emphasized that pregnant women may experience several episodes of malaria during pregnancy. The relationship between placental malaria, which is a direct consequence of malaria infection, and treatment outcomes for each malaria episode is not fully understood and may be heavily biased by gestational age at infection [105]. In this context, PCR-uncorrected ACPR, in which the half-life of drugs will play a key role, becomes an important indicator for choosing treatment options especially in high endemic settings [51]. Efficacy cannot be discussed separately from pregnancy outcomes as drugs may affect the fetus. Ideally, the safety of drugs should be assessed and reported within efficacy studies, using a standard which has been reviewed elsewhere [106]. Inclusion rather than exclusion of pregnant women into routine therapeutic efficacy studies could be a key change to address the paucity of data in the future when drugs have a clean teratogenicity profile. This would also permit comparison of efficacy and drug levels in pregnant and non-pregnant females. Recommendations specific to the determination of anti-malarial efficacy of uncomplicated $P$. falciparum infection in pregnancy beyond the WHO guideline for non-pregnant populations are listed in Table 2.

The assessment of anti-malarial efficacy presented here is limited by the paucity of data, the heterogeneity of studies, and the constraints of aggregated data meta-analysis. With no new RCTs on efficacy in pregnancy notified in trial registries, the treatment episodes in the cohort of studies described here will be the only data available in the near future to describe the efficacy of anti-malarials in pregnancy. Because of the paucity of studies, different methodologies and backgrounds (regional endemicity, seasonality of infection) of patients, conventional metaanalysis using aggregated data will not allow an optimal assessment of the findings. Reviews attempting aggregated data meta-analysis, including this work, should be interpreted with caution considering the different endpoints and drugs used. In addition, RCTs comparing ABT and QBT were mostly conducted in Southeast Asia. Though the conclusion of the comparison between ABT and QBT is unlikely to change considering the large magnitude of the effect, assessing smaller differences between ABTs would be valuable and cannot be effectively done
Table 2 Recommendations to determine antimalarial efficacy in uncomplicated $P$. falciparum infection in pregnancy (beyond current WHO standards for non-pregnant patients)

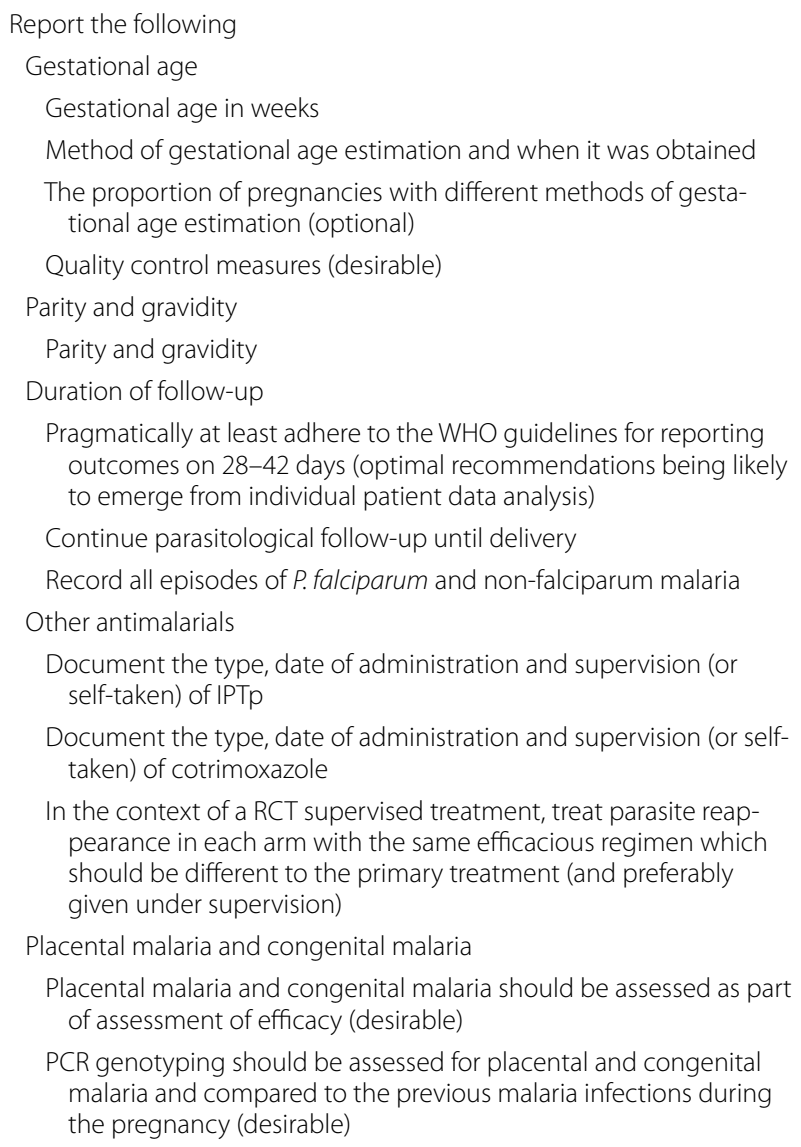

Placental malaria and congenital malaria should be assessed as part of assessment of efficacy (desirable)

PCR genotyping should be assessed for placental and congenital malaria and compared to the previous malaria infections during the pregnancy (desirable)

by aggregated data meta-analysis. Individual patient data meta-analyses offer a better methodological approach to summarize the currently available data, integrating the data from non-comparative studies. A new study group at the WorldWide Antimalarial Resistance Network [107] is proposed to tackle these issues by conducting individual patient data meta-analyses.

\section{Conclusions}

Although this and other reviews of ABTs and QBTs for malaria in pregnancy suggest ABTs are superior, further aggregated meta-analysis was hampered by inconsistencies in measurement and reporting. A standard framework for anti-malarial efficacy studies in pregnancy is warranted and will be a foundation for research with more comparable and reliable outputs. 


\section{Additional files}

Additional file 1. Search terms for literature review.

Additional file 3. Summary of the study design and reported outcomes.

Additional file 4. Summary of the quality of studies.

Additional file 5. Summary of treatment administration and interruption.

Additional file 6. The number of study arms with PCR-corrected outcomes for each comparison of drugs.

Additional file 7. Background information of patients at inclusion.

Additional file 8. Summary of gametocyte carriage.

Additional file 9. Funnel plots by quinine-based and artemisinin-based treatments.

Additional file 10. The PCR-uncorrected proportional adequate clinical and parasitological response (ACPR).

Additional file 11. Summary of evidence profile comparing artemisininbased and quinine-based treatment.

Additional file 12. Funnel plot of odds ratio of PCR-corrected treatment failure comparing quinine-based and artemisinin-based treatments.

Additional file 13. The methodology of assessing placental malaria.

\section{Abbreviations}

ABT: artemisinin-based treatments; AC: artesunate + clindamycin; ACPR: adequate clinical and parasitological response; AL: artemether-lumefantrine; ASAQ: artesunate-amodiaquine; ASMQ: artesunate-mefloquine; Cl: confidence interval; DP: dihydroartemisinin-piperaquine; IPTp: intermittent preventive treatment in pregnancy; PCR: polymerase chain reaction; PK: pharmacokinetic; QBT: quinine-based treatments; QC: quinine + clindamycin; RCT: randomized control trial; SP: sulfadoxine-pyrimethamine; WHO: World Health Organization.

\section{Authors' contributions}

MS and MEG did literature search. MS analysed the extracted information and wrote the first draft of the manuscript. MEG, FN, RM and PJG contributed significantly to the writing of the manuscript. All authors read and approved the final manuscript.

\section{Author details}

${ }^{1}$ WorldWide Antimalarial Resistance Network (WWARN), Oxford, UK. ${ }^{2}$ Centre for Tropical Medicine and Global Health, Nuffield Department of Medicine, University of Oxford, Old Road Campus, Roosevelt Drive, Oxford OX3 7FZ, UK. ${ }^{3}$ Shoklo Malaria Research Unit (SMRU), Mahidol-Oxford Tropical Medicine Research Unit, Faculty of Tropical Medicine, Mahidol University, Mae Sot, Tak, Thailand.

\section{Acknowledgements}

We would like to thank Prabin Dahal for his valuable advice particularly regarding statistical analyses. Finally, the authors thank all the healthcare workers and pregnant women who contributed to this body of research.

\section{Competing interests}

The authors declare that they have no competing interests.

\section{Availability of data and materials}

The extracted data will be available at WWARN website (http://www.wwarn. org/).

\section{Consent for publication}

Not applicable.

\section{Ethics approval and consent to participate}

Not applicable.

\section{Funding}

WWARN is supported by the Bill \& Melinda Gates Foundation. SMRU is part of the Mahidol Oxford University Research Unit supported by the Wellcome Trust of Great Britain. MS is currently supported by the University of Oxford Clarendon Fund. The funding bodies do not have roles in this review.

\section{Publisher's Note}

Springer Nature remains neutral with regard to jurisdictional claims in published maps and institutional affiliations.

Received: 23 October 2017 Accepted: 5 December 2017

Published online: 13 December 2017

\section{References}

1. Dellicour S, Tatem AJ, Guerra CA, Snow RW, ter Kuile FO. Quantifying the number of pregnancies at risk of malaria in 2007: a demographic study. PLoS Med. 2010;7:e1000221.

2. Desai M, ter Kuile FO, Nosten F, McGready R, Asamoa K, Brabin B, et al. Epidemiology and burden of malaria in pregnancy. Lancet Infect Dis. 2007:7:93-104

3. McGready R, Lee SJ, Wiladphaingern J, Ashley EA, Rijken MJ, Boel M, et al. Adverse effects of falciparum and vivax malaria and the safety of antimalarial treatment in early pregnancy: a population-based study. Lancet Infect Dis. 2012;12:388-96.

4. Moore KA, Fowkes FJI, Wiladphaingern J, Wai NS, Paw MK, Pimanpanarak $M$, et al. Mediation of the effect of malaria in pregnancy on stillbirth and neonatal death in an area of low transmission: observational data analysis. BMC Med. 2017;15:98.

5. Cottrell G, Moussiliou A, Luty AJ, Cot M, Fievet N, Massougbodji A, et al. Submicroscopic Plasmodium falciparum infections are associated with maternal anemia, premature births, and low birth weight. Clin Infect Dis. 2015;60:1481-8.

6. Rijken MJ, McGready R, Boel ME, Poespoprodjo R, Singh N, Syafruddin D, et al. Malaria in pregnancy in the Asia-Pacific region. Lancet Infect Dis. 2012;12:75-88.

7. Mayor A, Bardaji A, Macete E, Nhampossa T, Fonseca AM, Gonzalez R. Changing trends in P. falciparum burden, immunity, and disease in pregnancy. N Engl J Med. 2015;373:1607-17.

8. Clark RL. Embryotoxicity of the artemisinin antimalarials and potential consequences for use in women in the first trimester. Reprod Toxicol. 2009;28:285-96.

9. Li Q, Weina PJ. Severe embryotoxicity of artemisinin derivatives in experimental animals, but possibly safe in pregnant women. Molecules. 2010;15:40-57.

10. White TE, Clark RL. Sensitive periods for developmental toxicity of orally administered artesunate in the rat. Birth Defects Res B Dev Reprod Toxicol. 2008;83:407-17.

11. Clark RL, White TE, Clode SA, Gaunt I, Winstanley P, Ward SA. Developmental toxicity of artesunate and an artesunate combination in the rat and rabbit. Birth Defects Res B Dev Reprod Toxicol. 2004;71:380-94.

12. Clark RL, Arima A, Makori N, Nakata Y, Bernard F, Gristwood W, et al. Artesunate: developmental toxicity and toxicokinetics in monkeys. Birth Defects Res B Dev Reprod Toxicol. 2008;83:418-34.

13. Gomes C, Boareto AC, Dalsenter PR. Clinical and non-clinical safety of artemisinin derivatives in pregnancy. Reprod Toxicol. 2016;65:194-203.

14. WHO. Guidelines for the treatment of malaria. 3rd ed. Geneva: World Health Organization; 2015.

15. Nosten F, McGready R, d'Alessandro U, Bonell A, Verhoeff F, Menendez C, et al. Antimalarial drugs in pregnancy: a review. Curr Drug Saf. 2006;1:1-15.

16. Achan J, Talisuna AO, Erhart A, Yeka A, Tibenderana JK, Baliraine FN, et al. Quinine, an old anti-malarial drug in a modern world: role in the treatment of malaria. Malar J. 2011;10:144.

17. Nosten F, ter Kuile F, Thwai KL, Maelankirri L, White NJ. Spiramycin does not potentiate quinine treatment of falciparum malaria in pregnancy. Trans R Soc Trop Med Hyg. 1993;87:305-6. 
18. Moore KA, Simpson JA, Paw MK, Pimanpanarak M, Wiladphaingern J, Rijken MJ, et al. Safety of artemisinins in first trimester of prospectively followed pregnancies: an observational study. Lancet Infect Dis. 2016;16:576-83.

19. Mosha D, Mazuguni F, Mrema S, Sevene E, Abdulla S, Genton B. Safety of artemether-lumefantrine exposure in first trimester of pregnancy: an observational cohort. Malar J. 2014;13:197.

20. Dellicour S, Desai M, Aol G, Oneko M, Ouma P, Bigogo G, et al. Risks of miscarriage and inadvertent exposure to artemisinin derivatives in the first trimester of pregnancy: a prospective cohort study in western Kenya. Malar J. 2015;14:461.

21. Dellicour S, Sevene E, McGready R, Tinto H, Mosha D, Manyando C, et al. First-trimester artemisinin derivatives and quinine treatments and the risk of adverse pregnancy outcomes in Africa and Asia: a meta-analysis of observational studies. PLoS Med. 2017;14:e1002290.

22. WHO. Methods for surveillance of antimalarial drug efficacy. Geneva: World Health Organization; 2009.

23. Nosten F, Rogerson SJ, Beeson JG, McGready R, Mutabingwa TK, Brabin B. Malaria in pregnancy and the endemicity spectrum: what can we learn? Trends Parasitol. 2004;20:425-32.

24. Nosten F, McGready R, Mutabingwa T. Case management of malaria in pregnancy. Lancet Infect Dis. 2007;7:118-25.

25. Liberati A, Altman DG, Tetzlaff J, Mulrow C, Gotzsche PC, loannidis JP. The PRISMA statement for reporting systematic reviews and metaanalyses of studies that evaluate health care interventions: explanation and elaboration. PLoS Med. 2009;6:e1000100.

26. McGready $R$, Nosten F. Which drug is effective and safe for acute malaria in pregnancy? Reviewing the evidence. Drug Dev Res. 2010;71:56-68.

27. McGready R, White NJ, Nosten F. Parasitological efficacy of antimalarials in the treatment and prevention of falciparum malaria in pregnancy 1998 to 2009: a systematic review. BJOG. 2011;118:123-35.

28. Manyando C, Kayentao K, D'Alessandro U, Okafor HU, Juma E, Hamed K. A systematic review of the safety and efficacy of artemether-lumefantrine against uncomplicated Plasmodium falciparum malaria during pregnancy. Malar J. 2012;11:141.

29. Burger RJ, van Eijk AM, Bussink M, Hill J, Ter Kuile FO. Artemisinin-based combination therapy versus quinine or other combinations for treatment of uncomplicated Plasmodium falciparum malaria in the second and third trimester of pregnancy: a systematic review and meta-analysis. Open Forum Infect Dis. 2016;3:ofv170.

30. Brown LD, Cai TT, DasGupta A. Interval estimation for a binomial proportion. Stat Sci. 2001;16:101-17

31. Higgins JP, Thompson SG. Quantifying heterogeneity in a meta-analysis. Stat Med. 2002;21:1539-58.

32. Guyatt G, Oxman AD, Akl EA, Kunz R, Vist G, Brozek J, et al. GRADE guidelines: 1. Introduction-GRADE evidence profiles and summary of findings tables. J Clin Epidemiol. 2011;64:383-94.

33. McMaster University. GRADEpro GDT: GRADEpro Guideline Development Tool [Software]. 2015. https://gradepro.org. Accessed 10 Feb 2017.

34. Hunter JP, Saratzis A, Sutton AJ, Boucher RH, Sayers RD, Bown MJ. In meta-analyses of proportion studies, funnel plots were found to be an inaccurate method of assessing publication bias. J Clin Epidemiol. 2014;67:897-903.

35. Egger M, Davey Smith G, Schneider M, Minder C. Bias in meta-analysis detected by a simple, graphical test. Br Med J. 1997;315:629-34.

36. Naing T, Win H, Nwe YY. Falciparum malaria and pregnancy: relationship and treatment response. Southeast Asian J Trop Med Public Health. 1988;19:253-8.

37. Harinasuta T, Kietinun S, Somlaw SB, Somlaw SP, Bunnag D, Sheth UK, et al. A clinical trial of mefloquine on multi-resistant falciparum malaria in pregnant women in Thailand. Bulletin de la Societe Francaise de Parasitologie. 1990:419.

38. Sowunmi A, Oduola AM, Ogundahunsi OA, Fehintola FA, llesanmi OA, Akinyinka $\mathrm{OO}$, et al. Randomised trial of artemether versus artemether and mefloquine for the treatment of chloroquine/sufadoxinepyrimethamine-resistant falciparum malaria during pregnancy. J Obstet Gynaecol. 1998;18:322-7.

39. Bounyasong S. Randomized trial of artesunate and mefloquine in comparison with quinine sulfate to treat $P$. falciparum malaria pregnant women. J Med Assoc Thai. 2001;84:1289-99.
40. McGready R, Brockman A, Cho T, Cho D, van Vugt M, Luxemburger $C$, et al. Randomized comparison of mefloquine-artesunate versus quinine in the treatment of multidrug-resistant falciparum malaria in pregnancy. Trans R Soc Trop Med Hyg. 2000;94:689-93.

41. McGready R, Cho T, Samuel Villegas L, Brockman A, van Vugt M, et al. Randomized comparison of quinine-clindamycin versus artesunate in the treatment of falciparum malaria in pregnancy. Trans $\mathrm{R}$ Soc Trop Med Hyg. 2001;95:651-6.

42. McGready R, Ashley EA, Moo E, Cho T, Barends M, Hutagalung R, et al. A randomized comparison of artesunate-atovaquone-proguanil versus quinine in treatment for uncomplicated falciparum malaria during pregnancy. J Infect Dis. 2005;192:846-53.

43. Adam I, Ibrahim MH, Aelbasit IA, Elbashir MI. Low-dose quinine for treatment of chloroquine-resistant falciparum malaria in Sudanese pregnant women. East Mediterr Health J. 2004;10:554-9.

44. Kalilani L, Mofolo I, Chaponda M, Rogerson SJ, Alker AP, Kwiek JJ, et al. A randomized controlled pilot trial of azithromycin or artesunate added to sulfadoxine-pyrimethamine as treatment for malaria in pregnant women. PLoS ONE. 2007;2:e1166.

45. McGready R, Tan SO, Ashley EA, Pimanpanarak M, Viladpai-Nguen J, Phaiphun $L$, et al. A randomised controlled trial of artemether-lumefantrine versus artesunate for uncomplicated Plasmodium falciparum treatment in pregnancy. PLoS Med. 2008;5:e253.

46. Mutabingwa TK, Muze K, Ord R, Briceño M, Greenwood BM, Drakeley $C$, et al. Randomized trial of artesunate + amodiaquine, sulfadoxinepyrimethamine + amodiaquine, chlorproguanal-dapsone and SP for malaria in pregnancy in Tanzania. PLoS ONE. 2009;4:e5138.

47. Kaye DK, Nshemerirwe R, Mutyaba TS, Ndeezi G. A randomized clinical trial comparing safety, clinical and parasitological response to artemether-lumefantrine and chlorproguanil-dapsone in treatment of uncomplicated malaria in pregnancy in Mulago hospital, Uganda. J Infect Dev Ctries. 2008;2:135-9.

48. Piola P, Nabasumba C, Turyakira E, Dhorda M, Lindegardh N, Nyehangane $D$, et al. Efficacy and safety of artemether-lumefantrine compared with quinine in pregnant women with uncomplicated Plasmodium falciparum malaria: an open-label, randomised, non-inferiority trial. Lancet Infect Dis. 2010;10:762-9.

49. Carmona-Fonseca J, Agudelo-García OM, Arango-Flórez E. [Therapeutic efficacy and adverse events of treatments for vivax and falciparum malaria in pregnant women in the regions of Uraba and Alto San Jorge, Colombia, 2008-2011] (in Spanish). Rev Colomb Obstet Ginecol. 2013:64:27-37.

50. Pregact Study Group, Pekyi D, Ampromfi AA, Tinto H, Traoré-Coulibaly M, Tahita MC, et al. Four artemisinin-based treatments in African pregnant women with malaria. N Engl J Med. 2016;374:913-27.

51. Nambozi M, Kabuya J-BB, Hachizovu S, Mwakazanga D, Mulenga J, Kasongo W, et al. Artemisinin-based combination therapy in pregnant women in Zambia: efficacy, safety and risk of recurrent malaria. Malar J. 2017:16:199.

52. Osarfo J, Tagbor H, Cairns M, Alifrangis M, Magnussen P. Dihydroartemisinin-piperaquine versus artesunate-amodiaquine for treatment of malaria infection in pregnancy in Ghana: an open-label, randomised, non-inferiority trial. Trop Med Int Health. 2017;22:1043-52.

53. Onyamboko MA, Fanello Cl, Turner G, Jackson N, Tarning J, Nosten F, et al. Comparison of two regimens of artemether-lumefantrine for the treatment of uncomplicated Plasmodium falciparum malaria in pregnant women in the Democratic Republic of Congo. Am J Trop Med Hyg. 2015;93(4 Supplement):6.

54. Ukah M, Badejoko O, Ogunniyi S, Loto O, Aboderin O, Fatusi A. A randomized trial of artesunate-amodiaquine versus artemether-lumefantrine for the treatment of acute uncomplicated malaria in pregnancy. Int J Gynaecol Obstet. 2015;131:41-4.

55. Iribhogbe OI, Emmanuel I, Odianosen M. Comparative analysis of the safety and tolerability of fixed-dose artesunate/amodiaquine versus artemether/lumefantrine combinations for uncomplicated falciparum malaria in pregnancy: a randomized open label study. Clin Pharmacol. 2017;9:45-54

56. Anvikar A. Effective and safe treatment for malaria in pregnancy in India: a randomised controlled trial (CTRI/2009/091/001055). 2010.

57. McGready R. Randomised trial of 3 artemisinin combination therapy for malaria in pregnancy (NCT01054248). 2010. 
58. McGready R, Stepniewska K, Edstein MD, Cho T, Gilveray G, Looareesuwan $\mathrm{S}$, et al. The pharmacokinetics of atovaquone and proguanil in pregnant women with acute falciparum malaria. Eur J Clin Pharmacol. 2003;59:545-52.

59. Adam I, Tarning J, Lindegardh N, Mahgoub H, McGready R, Nosten F. Pharmacokinetics of piperaquine in pregnant women in Sudan with uncomplicated Plasmodium falciparum malaria. Am J Trop Med Hyg. 2012;87:35-40.

60. Onyamboko MA, Meshnick SR, Fleckenstein L, Koch MA, Atibu J, Lokomba V, et al. Pharmacokinetics and pharmacodynamics of artesunate and dihydroartemisinin following oral treatment in pregnant women with asymptomatic Plasmodium falciparum infections in Kinshasa DRC. Malar J. 2011;10:49.

61. McGready R, Phyo AP, Rijken MJ, Tarning J, Lindegardh N, Hanpithakpon $W$, et al. Artesunate/dihydroartemisinin pharmacokinetics in acute falciparum malaria in pregnancy: absorption, bioavailability, disposition and disease effects. Br J Clin Pharmacol. 2012;73:467-77.

62. Rijken MJ, McGready R, Phyo AP, Lindegardh N, Tarning J, Laochan N, et al. Pharmacokinetics of dihydroartemisinin and piperaquine in pregnant and nonpregnant women with uncomplicated falciparum malaria. Antimicrob Agents Chemother. 2011;55:5500-6.

63. Valea I, Tinto H, Traore-Coulibaly M, Toe LC, Lindegardh N, Tarning J, et al. Pharmacokinetics of co-formulated mefloquine and artesunate in pregnant and non-pregnant women with uncomplicated Plasmodium falciparum infection in Burkina Faso. J Antimicrob Chemother. 2014;69:2499-507.

64. Juma EA, Ogutu BR, Oloo F, Barwa T, Aman R. Pharmacokinetics of artemether-lumefantrine in pregnant and non-pregnant women with uncomplicated Plasmodium falciparum malaria in western Kenya. Am J Trop Med Hyg. 2014;91 (5 Supplement):587.

65. Mosha D, Guidi M, Mwingira F, Abdulla S, Mercier T, Decosterd LA, et al. Population pharmacokinetics and clinical response for artemetherlumefantrine in pregnant and nonpregnant women with uncomplicated Plasmodium falciparum malaria in Tanzania. Antimicrob Agents Chemother. 2014;58:4583-92.

66. Nyunt MM, Nguyen VK, Kajubi R, Huang L, Ssebuliba J, Kiconco S, et al. Artemether-lumefantrine pharmacokinetics and clinical response are minimally altered in pregnant Ugandan women treated for uncomplicated falciparum malaria. Antimicrob Agents Chemother. 2016;60:1274-82.

67. Mutagonda RF, Kamuhabwa AAR, Minzi OMS, Massawe SN, Maganda BA, Aklillu E. Malaria prevalence, severity and treatment outcome in relation to day 7 lumefantrine plasma concentration in pregnant women. Malar J. 2016;15:278.

68. Mutagonda RF, Kamuhabwa AAR, Minzi OMS, Massawe SN, Asghar M, Homann MV, et al. Effect of pharmacogenetics on plasma lumefantrine pharmacokinetics and malaria treatment outcome in pregnant women. Malar J. 2017;16:267.

69. Adam I, Idris HM, Elbashir MI. Quinine for chloroquine-resistant falciparum malaria in pregnant Sudanese women in the first trimester. East Mediterr Health J. 2004;10:560-5.

70. Adam I, Elwasila E, Mohammed Ali DA, Elansari E, Elbashir MI. Artemether in the treatment of falciparum malaria during pregnancy in eastern Sudan. Trans R Soc Trop Med Hyg. 2004;98:509-13.

71. Adegnika AA, Breitling LP, Agnandji ST, Chai SK, Schütte D, Oyakhirome $S$, et al. Effectiveness of quinine monotherapy for the treatment of Plasmodium falciparum infection in pregnant women in Lambaréné, Gabon. Am J Trop Med Hyg. 2005;73:263-6.

72. Adam I, Ali DM, Abdalla MA. Artesunate plus sulfadoxine-pyrimethamine in the treatment of uncomplicated Plasmodium falciparum malaria during pregnancy in eastern Sudan. Trans R Soc Trop Med Hyg. 2006;100:632-5.

73. Ndiaye JL, Ndiaye A, Faye B, Ba M, Tine R, Ndiaye D, et al. Open-label in vivo drug study to evaluate the safety and efficacy of artesunate plus amodiaquine combination in pregnant women with uncomplicated P. falciparum malaria in Senegal. Trop Med Int Health. 2011;16(Supplement 1):140.

74. Iribhogbe Ol, Igue EO, Odianosen M. Assessment of the safety of nonfixed-dose combination of artesunate and amodiaquine for uncomplicated falciparum malaria in pregnancy: a nonrandomized open-label study. J Pharm Health Serv Res. 2017;8:31-8.
75. McGready R, Cho T, Cho JJ, Simpson JA, Luxemburger C, Dubowitz L, et al. Artemisinin derivatives in the treatment of falciparum malaria in pregnancy. Trans R Soc Trop Med Hyg. 1998;92:430-3.

76. McGready R, Cho T, Hkirijaroen L, Simpson J, Chongsuphajaisiddhi T, White NJ, et al. Quinine and mefloquine in the treatment of multidrugresistant Plasmodium falciparum malaria in pregnancy. Ann Trop Med Parasitol. 1998;92:643-53.

77. McGready R, Cho T, Keo NK, Thwai KL, Villegas L, Looareesuwan S, et al. Artemisinin antimalarials in pregnancy: a prospective treatment study of 539 episodes of multidrug-resistant Plasmodium falciparum. Clin Infect Dis. 2001;33:2009-16.

78. Laochan N, Zaloumis SG, Imwong M, Lek-Uthai U, Brockman A, Sriprawat K, et al. Intervals to Plasmodium falciparum recurrence after anti-malarial treatment in pregnancy: a longitudinal prospective cohort. Malar J. 2015;14:221.

79. McGready R, Thwai KL, Cho T, Samuel Looareesuwan S, White NJ, et al. The effects of quinine and chloroquine antimalarial treatments in the first trimester of pregnancy. Trans R Soc Trop Med Hyg. 2002;96:180-4.

80. McGready R, Keo NK, Villegas L, White NJ, Looareesuwan S, Nosten F. Artesunate-atovaquone-proguanil rescue treatment of multidrugresistant Plasmodium falciparum malaria in pregnancy: a preliminary report. Trans R Soc Trop Med Hyg. 2003;97:592-4.

81. Villegas L, Hernandez N, Vasquez C, Veliz F, Guevara M-E, Salazar B, et al. Treatment of multi drug-resistant falciparum malaria during pregnancy with mefloquine-artesunate in Venezuela: preliminary results. Am J Trop Med Hyg. 2005;73(6 Supplement):225.

82. Rijken MJ, McGready R, Boel ME, Barends M, Proux S, Pimanpanarak M, et al. Dihydroartemisinin-piperaquine rescue treatment of multidrugresistant Plasmodium falciparum malaria in pregnancy: a preliminary report. Am J Trop Med Hyg. 2008;78:543-5.

83. Rulisa S, Kaligirwa N, Agaba S, Karema C, Mens PF, de Vries PJ. Pharmacovigilance of artemether-lumefantrine in pregnant women followed until delivery in Rwanda. Malar J. 2012;11:225.

84. Kalilani-Phiri L, Thesing PC, Nyirenda OM, Mawindo P, Madanitsa M, Membe $G$, et al. Timing of malaria infection during pregnancy has characteristic maternal, infant and placental outcomes. PLoS ONE. 2013;8:e74643.

85. Cohee LM, Kalilani-Phiri L, Mawindo P, Joshi S, Adams M, Kenefic L, et al. Parasite dynamics in the peripheral blood and the placenta during pregnancy-associated malaria infection. Malar J. 2016;15:483.

86. Ismail MR, Ordi J, Menendez C, Ventura PJ, Aponte JJ, Kahigwa E, et al. Placental pathology in malaria: a histological, immunohistochemical, and quantitative study. Hum Pathol. 2000;31:85-93.

87. Muehlenbachs A, Fried M, McGready R, Harrington WE, Mutabingwa TK, Nosten F, et al. A novel histological grading scheme for placental malaria applied in areas of high and low malaria transmission. J Infect Dis. 2010;202:1608-16.

88. Bulmer JN, Rasheed FN, Francis N, Morrison L, Greenwood BM. Placental malaria. I. Pathological classification. Histopathology. 1993;22:211-8.

89. Rogerson SJ, Mkundika P, Kanjala MK. Diagnosis of Plasmodium falciparum malaria at delivery: comparison of blood film preparation methods and of blood films with histology. J Clin Microbiol. 2003;41:1370-4.

90. Kloprogge F, Piola P, Dhorda M, Muwanga S, Turyakira E, Apinan S, et al. Population pharmacokinetics of lumefantrine in pregnant and nonpregnant women with uncomplicated Plasmodium falciparum malaria in Uganda. CPT Pharmacometrics Syst Pharmacol. 2013;2:e83.

91. McGready R, Stepniewska K, Lindegardh N, Ashley EA, La Y, Singhasivanon $\mathrm{P}$, et al. The pharmacokinetics of artemether and lumefantrine in pregnant women with uncomplicated falciparum malaria. Eur J Clin Pharmacol. 2006;62:1021-31.

92. Tarning J, Kloprogge F, Dhorda M, Jullien V, Nosten F, White NJ, et al. Pharmacokinetic properties of artemether, dihydroartemisinin, lumefantrine, and quinine in pregnant women with uncomplicated Plasmodium falciparum malaria in Uganda. Antimicrob Agents Chemother. 2013;57:5096-103.

93. Tarning J, McGready R, Lindegardh N, Ashley EA, Pimanpanarak M, Kamanikom B, et al. Population pharmacokinetics of lumefantrine in pregnant women treated with artemether-lumefantrine for uncomplicated Plasmodium falciparum malaria. Antimicrob Agents Chemother. 2009;53:3837-46. 
94. Hutagalung R, Paiphun L, Ashley EA, McGready R, Brockman A, Thwai $\mathrm{KL}$, et al. A randomized trial of artemether-lumefantrine versus mefloquine-artesunate for the treatment of uncomplicated multi-drug resistant Plasmodium falciparum on the western border of Thailand. Malar J. 2005;4:46.

95. Vugt MV, Wilairatana P, Gemperli B, Gathmann I, Phaipun L, Brockman A, et al. Efficacy of six doses of artemether-lumefantrine (benflumetol) in multidrug-resistant Plasmodium falciparum malaria. Am J Trop Med Hyg. 1999;60:936-42.

96. Woodrow CJ, White NJ. The clinical impact of artemisinin resistance in Southeast Asia and the potential for future spread. FEMS Microbiol Rev. 2017:41:34-48.

97. Moore KA, Simpson JA, Wiladphaingern J, Min AM, Pimanpanarak M Paw MK, et al. Influence of the number and timing of malaria episodes during pregnancy on prematurity and small-for-gestational-age in an area of low transmission. BMC Med. 2017;15:117.

98. Rogerson SJ, Hviid L, Duffy PE, Leke RF, Taylor DW. Malaria in pregnancy: pathogenesis and immunity. Lancet Infect Dis. 2007;7:105-17.

99. Costantine MM. Physiologic and pharmacokinetic changes in pregnancy. Front Pharmacol. 2014:5:65

100. Ward SA, Sevene EJ, Hastings IM, Nosten F, McGready R. Antimalarial drugs and pregnancy: safety, pharmacokinetics, and pharmacovigilance. Lancet Infect Dis. 2007;7:136-44.

101. Stepniewska K, Taylor WR, Mayxay M, Price R, Smithuis F, Guthmann JP, et al. In vivo assessment of drug efficacy against Plasmodium falciparum malaria: duration of follow-up. Antimicrob Agents Chemother. 2004:48:4271-80
102. Mayor A, Serra-Casas E, Bardají A, Sanz S, Puyol L, Cisteró P, et al. Submicroscopic infections and long-term recrudescence of Plasmodium falciparum in Mozambican pregnant women. Malar J. 2009;8:9.

103. Stepniewska K, White NJ. Some considerations in the design and interpretation of antimalarial drug trials in uncomplicated falciparum malaria. Malar J. 2006;5:127.

104. Moore KA, Simpson JA, Thomas KH, Rijken MJ, White LJ, Lu Moo Dwel $S$, et al. Estimating gestational age in late presenters to antenatal care in a resource-limited setting on the Thai-Myanmar border. PLoS ONE. 2015;10:e0131025.

105. McGready R, Davison BB, Stepniewska K, Cho T, Shee H, Brockman A, et al. The effects of Plasmodium falciparum and $P$. vivax infections on placental histopathology in an area of low malaria transmission. Am J Trop Med Hyg. 2004;70:398-407.

106. Saito M, Gilder ME, Nosten F, Guérin PJ, McGready R. Methodology of assessment and reporting of safety in anti-malarial treatment efficacy studies of uncomplicated falciparum malaria in pregnancy: a systematic literature review. Malar J. 2017. https://doi.org/10.1186/ s12936-017-2136-x

107. Malaria in Pregnancy Treatment Efficacy Study Group (WorldWide Antimalarial Resistance Network). Assessing the efficacy of a range of antimalarials used for the treatment of $P$. falciparum malaria in all trimesters of pregnancy in Africa and Asia. 2016. http://www.wwarn. org/working-together/study-groups/malaria-pregnancy-treatmentefficacy-study-group. Accessed 10 Feb 2017.

\section{Submit your next manuscript to BioMed Central and we will help you at every step:}

- We accept pre-submission inquiries

- Our selector tool helps you to find the most relevant journal

- We provide round the clock customer support

- Convenient online submission

- Thorough peer review

- Inclusion in PubMed and all major indexing services

- Maximum visibility for your research

Submit your manuscript at www.biomedcentral.com/submit
() Biomed Central 